INVE STIGACIÓN DIDÁCTICA

\title{
Hombres y mujeres ante su formación universitaria. Expectativas de éxito en estudios científico-tecnológicos y barreras percibidas
}

\author{
Carmen María Fernández García, Susana Torío López Universidad de Oviedo* \\ y Omar García Pérez
}

RESUMEN: Existen diversos factores que condicionan las elecciones académicas y profesionales de los estudiantes, así como sus creencias de autoeficacia en el afrontamiento de su formación. El presente artículo analiza algunos de estos factores con una amplia muestra de estudiantes universitarios de Grado de estudios científicos y tecnológicos. Los resultados indican algunas diferencias de comportamiento entre los diversos Grados, un optimismo generalizado en sus expectativas de éxito a pesar de reconocer que tendrán ciertas dificultades de comprensión así como, en algunos casos, una percepción de haber experimentado alguna discriminación por motivo de género.

Palabras clave: Género; Ciencia; Estudios universitarios; Autoeficacia.

ABSTRACT: Men and women towards higher education: expectations of success in scientific-technological studies and perceived barriers.

Students' academic and vocational choices and their self-efficacy beliefs in coping with their training are influenced by several factors. This article discusses some of these factors in a sample of students of Higher Education who are studying science and technology studies. The results show some differences in behaviour between the different Bachelor Degrees and a general optimism in their expectations of success. In spite of this, the students expect to have some difficulties in comprehension and, in some cases, they recognise having experienced certain discrimination based on their gender.

KeYwords: Gender; Science; Higher Education; Self- efficacy.

RÉSUMÉ: Hommes et femmes devant sa formation universitaire. Expectatives de succès dans des études scientifique-technologiques et les barriers perçues.

II y a plusieurs facteurs qui influencent les choix scolaires et professionnels des étudiants aussi bien que leurs croyances sur l'auto-efficacité pour faire face à leur formation. Cet article analyse certains de ces facteurs dans un large échantillon d'étudiants universitaires de Licence en études scientifiques et technologiques. Les résultats montrent quelques différences de comportement entre les divers Licences, un optimisme généralisé dans leurs attentes de succès, tout en reconnaissant qu'ils auront des difficultés de compréhension, et dans certains cas, la perception d'avoir été victimes de quelque sorte de discrimination de genre.

Mots clé: Genre; Science; Études universitaires; Auto-efficacité.

\footnotetext{
"Facultad de Formación de Profesorado y Educación. Universidad de Oviedo. Dirección de contacto: Carmen María Fernández García. Departamento de Ciencias de la Educación. C/ Aniceto Sela s/n, Despacho 235. 33005 Oviedo (España). Correo electrónico: fernandezcarmen@uniovi.es $\triangle$ Artículo recibido el 18 de Marzo de 2013 y aceptado el 24 de Enero de 2014.
} 


\section{Introducción}

En los últimos años se han desarrollado numerosos estudios e investigaciones para trata de determinar las razones que llevan a los estudiantes de enseñanza secundaria postobligatoria a elegir unos determinados estudios universitarios frente a otros, las especialidades por las que se muestra una mayor preferencia o e rendimiento obtenido en las diversas materias.

El Grupo de Investigación ASOCED (Grupo de Análisis Sociológico y Cultural de los Procesos Escolares y Educativos) es partícipe de este interés por conocer los argumentos en los que los estudiantes se basan para tomar este tipo de decisiones, emprendiendo para ello diversas investigaciones (Fernández et al., 2006 Rodríguez-Menéndez et al., 2006; Fernández et al., 2007; López et al., 2007).

En la actualidad, el grupo trata de determinar las diferencias de género que pueden estar mediando en estos itinerarios mediante una investigación que estudia la influencia de la autoeficacia en el rendimiento académico y en la elección de estudios científico-tecnológicos de los estudiantes de secundaria y universidad. Se trata de un proyecto financiado por el $\mathrm{Mi}$ nisterio de Ciencia e Innovación iniciado en el año 2010 y que finalizará en el año 2013. En el presente artículo nos centraremos únicament en el análisis de los datos obtenidos a partir de las respuestas de los estudiantes de la Universidad focalizando nuestra atención de manera particular, en la variable que denominaremo "persuasión y apoyo social". De manera más concreta, se pretende: 1) analizar las expectativas de éxito de los estudiantes; 2) profundizar en el influjo de la variable que denominaremos "persuasión y apoyo social" y 3) conocer la influencia de la variable género en todo ello.

\section{Género, estudios universitarios y futura salida profesional}

Uno de los elementos que puede condicionar las elecciones de los estudiantes en su acceso a la Universidad, en las materias opta- tivas por las que se inclinan o incluso en las futuras elecciones profesionales, es la creencia de que existen diferencias de género en la habilidad o facilidad para el aprendizaje y desempeño en determinadas actividades. Una de las más extendidas es la que considera que los hombres tienen una dotación "natural" para las ciencias o las matemáticas mientras, por el contrario, las mujeres la tendrían para las actividades vinculadas con el lenguaje o las cuestiones de orden social. Sin embargo, como han planteado las revisiones realizadas por algunos estudios (Erwin y Maurutto, 1998; Guimond y Roussel, 2001; Martín-Del Buey y Camarero-Suárez, 2001) esta afirmación no se sustenta en todas las investigaciones empíricas desarrolladas recientemente. Incluso puede afirmarse que no existiría tal competencia o incompetencia entendida de una forma general sino que, ya desde edades tempranas, puede constatarse que dentro de la competencia científica hay cualidades en las que los hombres obtienen mejores resultados mientras que en otras dimensiones son las mujeres las que se ubican en las primeras posiciones (MarbáTallada y Solsona-Pairó, 2012). Parece además que estas diferencias se explicarían más por el proceso de socialización diferenciado que han vivido cada uno de los géneros, los valores que el sujeto pueda ir incorporando en su trayectoria vital - escolar o aspectos motivadores/ desmotivadores ante determinadas tareas, que por verdaderas diferencias cognitivas explicables mediante factores puramente biológicos (Ayalon, 2003; Bermúdez et al., 2011; BlättelMink, 2002; Evers et al., 2006; García-Gómez et al., 2009; Marbá-Tallada y Solsona-Pairó, 2012; Martín-Del Buey y Camarero-Suárez, 2001; Rodd y Bartholomew, 2006; Rego y Castro, 2002; Sax y Harper, 2007). Por lo tanto, se concluye que este proceso de socialización es construido desde las diversas esferas en las que los sujetos se desenvuelven: su ambiente familiar más próximo, el influjo de los iguales, la repercusión de las instituciones académicas y sus docentes, los mensajes que la sociedad transmite a través de sus medios de comunicación, etc
En todo caso, con independencia de esta comprobación parece necesario tener muy en mujeres se encuentran más presionadas y obtienen peores resultados cuando se encuentran preocupadas por confirmar el estereotipo que las sitúa como peor dotadas para lo científicomatemático. Así, llegan incluso en ocasiones considerar que si obtienen mejores resultados que los varones en estos ámbitos, es debido factores externos tales como que el nivel de 2002; Guimond y Roussel, 2001), que poseen una gran memoria -no se menciona la habilidad- (Rodd y Bartholomew, 2006) o el convencimiento de que son menos capaces que sus compañeros varones (Erwin y Maurutto, 1998 Guimond y Roussel, $2001^{1}$ ) aun cuando sus resultados no corroboren esta percepción. Por lo tanto, en algunos casos, los varones y las mujeres con un fuerte estereotipo de género suelen percibirse como más dotados para las ciencias o lengua respectivamente (Guimond y Roussel, 2001) que aquéllos que no poseen un estereotipo de género tan intenso.

La teoría social cognitiva de la carrera desarrollada por Lent y colaboradores trabaja esto condicionantes y establece que las creencias de autoeficacia son junto con otras variables, de cisivas en la elección vocacional. Así, el interés por la materia, las expectativas de resultados las metas o intentos para persistir en una actividad o el apoyo/barreras sociales serían de terminantes en el proceso de decisión acadé mica y vocacional. Por expresarlo de un modo más clarificador, sería posible afirmar que las personas forman sus creencias de autoeficacia interpretando la información que obtienen de cuatro fuentes o recursos fundamentales (Betz y Borgen, 2000; Britner y Pajares, 2006; Hacket y Betz, 1981; Lent et al., 1996; Miura, 1987; Pajares, 2005; Zeldin y Pajares, 2000; Zeldin et al. 2008). Éstas serían las siguientes: cuenta esta creencia "biologicista" pues, las exigencia en su caso es inferior (Blättel-Mink

1. Experiencias previas: los estudiantes realizan tareas e interpretan los resultados de sus acciones, y usan esta información para desarrollar creencias sobre su competencia para el afrontamiento de tareas similares. Normalmente, experiencias previas de éxito ayudan a incrementar la autoeficacia; al contrario, las que conducen al fracaso provocan una disminución de la autoeficacia.

2. Modelado y aprendizaje vicario: los estudiantes también forman sus creencias de autoeficacia observando a otros mientras realizan dichas tareas y comparando su propia competencia con la de esas personas. Para que este recurso ejerza su influencia, el modelo observado debe poseer características personales similares a las del observador. De este modo, la persona puede imaginar que "si él/ella puede hacerlo, yo también soy capaz".

3. Persuasión y apoyo social: este recurso supone la exposición a los juicios, verbales y no verbales de otras personas significativas para el individuo. La persuasión social positiva puede ayudar a aumentar las creencias sobre la propia competencia y, al contrario, los refuerzos y estímulos negativos pueden desarrollar el sentido de la propia incapacidad. Puesto que la sociedad ha definido los patrones de conducta socialmente deseables para cada uno de los sexos, varones y mujeres reciben mensajes positivos y negativos cuando realizan determinadas elecciones académicas. Estos mensajes provienen del entorno social y son los progenitores, docentes y amigos/amigas quienes ejercen la mayor influencia a través de los mecanismos de persuasión social.

4. Estado de arousal: el estado emocional que experimenta una persona cuando contempla o realiza una tarea influye en la formación de las creencias de autoeficacia. Si hay estrés y ansiedad, estas creencias pueden ser bajas, mientras que serán altas si la persona está relajada.

1 Paradójicamente en este mismo estudio se observa que los varones, sin embargo, consideran que la dotación de hombres y mujeres para las ciencias es igual (Guimond y Roussel, 2001). Esta misma apreciación en cuanto a la igualdad o preferencia de hombres y mujeres por las matemáticas se pone de manifiesto en Rodd y Bartholomew (2006). 
Por tanto, las personas elaboramos nuestras creencias de autoeficacia a través de la interpretación de la información que recibimos de los cuatro recursos mencionados. La relevancia de cada uno de ellos en la formación de tales creencias dependerá de la materia o actividad y del proceso de integración que efectúe cada persona (Pajares, 2005; Zeldin et al., 2008). Así, por ejemplo, en lo que respecta a las matemáticas, se ha demostrado que las experiencias previas, la persuasión socia y el estado de arousal correlacionan entre ellos de forma muy significativa. En cuanto al apren dizaje vicario, se concluyó que estaba moderadamente relacionado con la persuasión social $y$ tenía una correlación muy baja con las otras do variables (Lent, López, Brown y Gore, 1996).

En este contexto, diversos autores (Bandura et al., 2001; Betz y Hackett, 1981, 1997, 2006; Betz y Schifano, 2000; Hackett, 1985; Hackett y Betz, 1981; Olaz, 2003; Vouillot et al., 2004 Zeldin y Pajares, 2000) han indicado que las creencias de autoeficacia tienen un peso especí fico muy importante para explicar el desarrollo de la carrera de las mujeres. Se postula que no desarrollan altas creencias de autoeficacia para las disciplinas científico-tecnológicas y este hecho explica su menor presencia en ellas.

\section{Material y método}

\section{Muestra}

En la investigación han participado 813 estudiantes que cursan su segundo año de carrer en distintas especialidades de los Grados de Ingeniería y estudios de Ciencias en la Universidad de Oviedo (289 chicas y 522 chicos, lo que representa un $36 \%$ y $64 \%$ del total de la muestra respectivamente). Sus edades se encuentran comprendidas entre los 18 y 37 años $(M e$ dia=19,72 y D.T.=1.9). La variable edad tení una asimetría negativa y era leptocúrtica, por lo que se consideró emplear la prueba $U$ de ManWhitney para determinar si había diferencia estadísticamente significativas entre chicos chicas. Los resultados mostraron que existían tales diferencias $(\mathrm{U}=54336,5, \mathrm{p}=0.001)$.
En las chicas, la edad media es de 19 años con una D.T.=1.77; en los chicos la edad media es de 20 años con una D.T.=2. El rango de edad en las mujeres oscila entre los 18 y 29 años, mientras que en los hombres el rango es de 18 hasta 37 años.

La distribución por género y estudios muestra (Tabla 1) una relación estadísticamente significativa entre ambas $(\chi 2=319,44, p=0.00$; Coeficiente de Contingencia $=0.53, p=0.00$ ). En casi todas las especialidades son mayoría los chicos, menos en Química Industrial, donde las chicas les doblan en número (68.4\% frente a 31.6\%), Biotecnología (57.9\% frente a 42,1\%), Química ( $54.3 \%$ frente a $45.7 \%$ ), Biología ( $57.8 \%$ y $42.2 \%$ ) y en Matemáticas donde las chicas llegan casi a cuadriplicar a sus compañeros varones $(79.1 \%$ frente a 20.9\%). La presencia masculina es especialmente patente en la mayoría de las especialidades de Ingeniería y de manera muy particular en Informática en Tecnologías de la Información (93,5\%), Informática del Software (87\%) e Ingeniería Forestal y del Medio Natural (85,7\%).

A efectos de algunos de análisis estadísticos se ha categorizado en algún caso la muestra en dos grandes grupos: Ingenierías y Ciencias. Dentro de la primera se incluirían los estudiantes de las diversas especialidades de Tecnología o de Ingeniería y dentro de la segunda los estudios de Geología, Biotecnología, Química, Física, Biología y Matemáticas. La fragmentación de la muestra en estos dos grupos obedece a razones que han sido fruto de un análisis y reflexión riguroso. En efecto, los estudios estadísticos iniciales realizados como aproximación exploratoria a la muestra, han permitido concluir que existen importantes variaciones entre estos dos grupos en cuanto a sus preferencias profesionales y expectativas académicas

\section{Procedimiento e instrumentos}

En el proceso de recogida de información de los estudiantes correspondientes a la muestra universitaria, estos completaron un cuestionario durante una clase del primer semestre del curso académico. Este cuestionario, que contestaron de

\begin{tabular}{|l|l|l|l|}
\cline { 2 - 4 } \multicolumn{1}{c|}{} & \multicolumn{1}{c|}{$\begin{array}{c}\text { CHICAS } \\
\text { (N=163) }\end{array}$} & $\begin{array}{c}\text { CHICOS } \\
(\mathrm{N}=4 \mathbf{4 1 6})\end{array}$ & TOTAL \\
\hline Eléctrica & $7(29.2 \%)$ & $17(70.8 \%)$ & $24(2.9 \%)$ \\
\hline Electrónica Industrial y Automática & $9(25 \%)$ & $27(75 \%)$ & $36(4.4 \%)$ \\
\hline Química Industrial & $13(68.4 \%)$ & $6(31.6 \%)$ & $19(2.3 \%)$ \\
\hline Mecánica & $23(24.5 \%)$ & $71(75.5 \%)$ & $94(11.6 \%)$ \\
\hline Tecnologías Industriales & $22(25 \%)$ & $66(75 \%)$ & $88(10.8 \%)$ \\
\hline Tecnología y Servicios de Telecomunicación & $21(32.3 \%)$ & $44(67.4 \%)$ & $65(7.9 \%)$ \\
\hline Informática en Tecnologías de la Información & $2(6.5 \%)$ & $29(93.5 \%)$ & $31(3.8 \%)$ \\
\hline Tecnología Mineras & $19(38.8 \%)$ & $30(61.2 \%)$ & $49(6 \%)$ \\
\hline Informática del Software & $10(13 \%)$ & $67(87 \%)$ & $77(9.5 \%)$ \\
\hline Recursos Mineros y Energéticos & $15(38.5 \%)$ & $24(61.5 \%)$ & $39(4.8 \%)$ \\
\hline Geomática y Topografía & $9(45 \%)$ & $11(55 \%)$ & $20(2.5 \%)$ \\
\hline Forestal y del Medio Natural & $2(14.3 \%)$ & $12(85.7 \%)$ & $14(1.7 \%)$ \\
\hline Ingeniería Química & $11(47.8 \%)$ & $12(52.2 \%)$ & $23(2.8 \%)$ \\
\hline Geología & $11(47.8 \%)$ & $12(52.2 \%)$ & $23(2.8 \%)$ \\
\hline Biotecnología & $11(57.9 \%)$ & $8(42.1 \%)$ & $19(2.3 \%)$ \\
\hline Química & $25(54.3 \%)$ & $21(45.7 \%)$ & $46(5.6 \%)$ \\
\hline Física & $8(26.7 \%)$ & $22(73.3 \%)$ & $30(3.7 \%)$ \\
\hline Biología & $52(57.8 \%)$ & $38(42.2 \%)$ & $90(11 \%)$ \\
\hline Matemáticas & $19(79.1 \%)$ & $5(20.9 \%)$ & $24(2.9 \%)$ \\
\hline
\end{tabular}

Tabla 1. Distribución por género y estudios.

manera voluntaria y anónima, fue administrado personalmente por el equipo de investigación.

El cuestionario constaba de un total de 77 ítems y ha sido el resultado de una traducción y adaptación a la población española del instrumento Engineering Fields Questionnaire. La versión original del instrumento fue solicitada y posteriormente enviada al equipo por el propio autor, Robert W. Lent de la Universidad de Maryland. Antes de proceder a su aplicación, se realizó el proceso de traducción del idioma inglés al español, tomándose las medidas oportunas para adaptar expresiones lingüísticas del inglés americano que no están presentes en la cultura académica española. Cabe resaltar que, si bien inicialmente se decidió usar la metodología «backward translation», realizando la traducción del inglés al español y traduciendo la nueva versión castellana al inglés, sin embargo, debido a que los autores del instrumento original no dominaban el idioma español, fue necesario reconsiderar esta decisión, optando por un proceso de traducción «forward translation».

Este instrumento permite medir variables como: creencias de autoeficacia (5 items), expectativas de resultado (10 items), interés por los estudios cursados (2 items), metas (10 items), barreras y apoyos sociales (17 items). 
Para medir las creencias de autoeficacia se incluyó una subescala ( $1=$ nada de confianza a $9=$ absoluta confianza) que preguntaba por su grado de confianza para cursar con éxito lo estudios que estaban realizando (Coeficiente $\alpha=.88)$. Para medir las expectativas de resultado, se incluyeron ítems en los que se indagaba sobre la opinión que tenían acerca de la relevancia de los estudios elegidos para sus planes profesionales (escala 1 a 9) (Coeficiente $\alpha=.88$ ). La variable "interés por los estudios cursados" fue medida con ítems referidos al interés por estudiar determinados tópicos y realizar actividades de ciencia y tecnología (escala 1 a 5 ) (Coeficiente $\alpha=.82$ ). Para la variable "metas", se introdujeron ítems para medir los intentos por persistir en los estudios elegidos (escala a 5) (Coeficiente $\alpha=.88$ ). Las barreras y apoyo sociales fueron medidos mediante ítems en lo que debían indicar el refuerzo que obtenían de distintos elementos que facilitaban/dificultaban su trayectoria formativa (escala 1 a 5 ) (Coeficiente $\alpha=.82$ ). Para una información más exhaustiva sobre la estructura interna del instrumento y validación del instrumento pued consultarse Rodríguez-Menéndez et al. (2015).

\section{Análisis de Dato}

En el presente artículo nos hemos centrado en el análisis de algunos de los ítems que ofrecen información directa sobre los tres objetivo

principales del estudio mediante el programa estadístico SPSS. Así, se ha realizado análisis de frecuencias para conocer la distribución de la muestra en los distintos ítems, prestando especial atención a las variaciones entre estudiantes de Ciencias e Ingeniería. También se han llevado a cabo análisis de contingencias para verificar si existían diferencias significativas entre algunos de los ítems y la variable género.

\section{Resultados}

Una primera cuestión interesante para nuestro análisis es el grado de optimismo manifestado por los estudiantes en relación a sus posibilidades de éxito en los estudios. Como puede apreciarse en la Tabla 2 salvo un porcentaje muy ajustado de alumnos la mayoría de los estudiantes confían en permanecer matriculados en este curso, destacando que la mayoría de los estudiantes $(88.7 \%)$ se ubican en la categoría que más fehacientemente considera que continuará matriculado en sus estudios. Apreciamos, por tanto, que parece haber una diferencia notable respecto a la situación que la literatura pedagógica afirma que se produce en el primer curso de los estudios universitarios y, por la cual, un buen número de estudiantes se plantean muy seriamente la posibilidad de cambiar de estudios o incluso abandonar por completo su formación universitaria (Feldman, 2005). Una situación bastante semejante se aprecia cuando tratamos

\begin{tabular}{|c|c|c|c|c|c|c|c|c|c|c|}
\hline \multirow{3}{*}{$\begin{array}{l} \\
\text { Pienso } \\
\text { permanecer } \\
\text { matriculado en } \\
\text { este Grado }\end{array}$} & \multicolumn{2}{|c|}{$\begin{array}{l}\text { TOTALMENTE EN } \\
\text { DeSACUERDo }\end{array}$} & \multicolumn{2}{|c|}{$\begin{array}{l}\text { Más bien en } \\
\text { Desacuerdo }\end{array}$} & \multicolumn{2}{|c|}{$\begin{array}{c}\text { No estoy } \\
\text { Seguro }\end{array}$} & \multicolumn{2}{|c|}{ De ACuerdo } & \multicolumn{2}{|c|}{$\begin{array}{c}\text { TOTALMENTE DE } \\
\text { ACUERDO }\end{array}$} \\
\hline & \multicolumn{2}{|c|}{$3(0.4 \%)$} & \multicolumn{2}{|c|}{$2(0.2 \%)$} & \multicolumn{2}{|c|}{$15(1.8 \%)$} & \multicolumn{2}{|c|}{$71(8.7 \%)$} & \multicolumn{2}{|c|}{$721(88.7 \%)$} \\
\hline & $\begin{array}{l}\text { I } \\
3\end{array}$ & $\begin{array}{l}\mathrm{C} \\
-\end{array}$ & $\begin{array}{l}\text { I } \\
2\end{array}$ & $\begin{array}{l}\mathrm{C} \\
-\end{array}$ & $\begin{array}{l}\mathbf{I} \\
12\end{array}$ & $\begin{array}{l}\mathrm{C} \\
3\end{array}$ & $\begin{array}{c}\text { I } \\
53\end{array}$ & $\begin{array}{l}\text { C } \\
18\end{array}$ & $\begin{array}{c}\text { I } \\
510\end{array}$ & $\begin{array}{c}\mathrm{C} \\
211\end{array}$ \\
\hline \multirow{2}{*}{$\begin{array}{l}\text { Pienso que } \\
\text { conseguir } \\
\text { el Grado es } \\
\text { un objetivo } \\
\text { realista para mí }\end{array}$} & \multicolumn{2}{|c|}{$1(0.1 \%)$} & \multicolumn{2}{|c|}{$2(0.2 \%)$} & \multicolumn{2}{|c|}{$34(4.2 \%)$} & \multicolumn{2}{|c|}{$185(22.8 \%)$} & \multicolumn{2}{|c|}{$590(72.7 \%)$} \\
\hline & $\begin{array}{l}\text { I } \\
1\end{array}$ & $\begin{array}{l}\mathrm{C} \\
-\end{array}$ & $\begin{array}{l}\text { I } \\
2\end{array}$ & $\begin{array}{l}\mathrm{C} \\
-\end{array}$ & $\begin{array}{c}\mathbf{I} \\
27\end{array}$ & $\begin{array}{l}\mathrm{C} \\
7\end{array}$ & $\begin{array}{c}\mathbf{I} \\
131\end{array}$ & $\begin{array}{l}\mathrm{C} \\
54\end{array}$ & $\begin{array}{c}\mathbf{I} \\
419\end{array}$ & $\begin{array}{c}\mathbf{C} \\
171\end{array}$ \\
\hline
\end{tabular}

Tabla 2. Optimismo de los estudiantes respecto a su continuidad en el Grado. de determinar si se consideran capaces de finalizar sus estudios universitarios: solamente un $0.1 \%$ manifiestan estar «Totalmente en desacuerdo», un $0.2 \%$ «Más bien en desacuerdo» «No están seguros» un $4.2 \%$.

En la Tabla 2 se presentan además desglosadas las respuestas de los dos grandes grupo en los que se ha dividido a los estudiantes: Ingeniería (I) y Ciencias (C). Como puede apreciarse, son siempre estudiantes del primero de los grupos los que se ubican en las opciones de respuesta que suponen que tendrán mayore dificultades tanto en continuar sus estudio durante ese semestre como en ser capaces de culminarlos. Igualmente, dentro de cada uno de los grupos las respuestas más optimistas son ligeramente superiores en los estudiantes $\mathrm{d}$ ciencias. Estos datos nos ofrecen pistas que será necesario explorar con mayor profundidad en el momento en el que se emprenda la fase cua- litativa de la investigación. En efecto, conviene estudiar exhaustivamente los apoyos que perciben los estudiantes -y especialmente los de ingenierías- procedentes de su ambiente familiar o del entorno académico.

En todo caso, dados los antecedentes encontrados en la revisión de la literatura pedagógica dizar en la variable género, encontrando datos sorprendentes. A saber, en toda la muestra se observa una relación estadísticamente significativa (Coeficiente de Contingencia $=0.332, \mathrm{p}=$ 0.000 ) entre el ítem referido a terminar el Grado y otro pregunta del instrumento en el que se hacía referencia a si los estudiantes se «sienten diferentes al resto por motivo de género» (Ver Tabla 3). Más aún, también se aprecia esta relación entre su expectativa de finalizar los estudios y su consideración de que han recibido un trato injusto por su género en los primeros sobre este particular, hemos querido profun-

\begin{tabular}{|c|c|c|c|c|c|c|c|c|c|c|c|}
\hline & & Posi & LIDA & $\mathrm{SEl}$ & RME & ERE & $\mathrm{AL}$ & $\mathrm{OP}$ & мO1 & $\mathrm{O} \mathrm{DE}$ & NERO \\
\hline & & & una & & & & & & & $\mathrm{Mu}$ & sible \\
\hline \multirow{10}{*}{$\begin{array}{l}\text { Pienso que } \\
\text { conseguir } \\
\text { el Grado es } \\
\text { un objetivo } \\
\text { realista } \\
\text { para mí }\end{array}$} & \multirow[b]{2}{*}{$\begin{array}{l}\text { Totalmente en } \\
\text { desacuerdo }\end{array}$} & \multicolumn{2}{|c|}{0} & \multicolumn{2}{|c|}{0} & \multicolumn{2}{|c|}{$1(100 \%)$} & \multicolumn{2}{|c|}{0} & \multicolumn{2}{|c|}{0} \\
\hline & & $\begin{array}{l}I \\
-\end{array}$ & C & I & $\begin{array}{l}\mathrm{C} \\
-\end{array}$ & $\begin{array}{l}\mathrm{I} \\
1\end{array}$ & C & I & $\begin{array}{l}\mathrm{C} \\
-\end{array}$ & I & $\begin{array}{l}\mathrm{C} \\
-\end{array}$ \\
\hline & \multirow{2}{*}{$\begin{array}{l}\text { Más bien en } \\
\text { desacuerdo }\end{array}$} & \multicolumn{2}{|c|}{$1(50 \%)$} & \multicolumn{2}{|c|}{0} & \multicolumn{2}{|c|}{0} & \multicolumn{2}{|c|}{0} & \multicolumn{2}{|c|}{$1(50 \%)$} \\
\hline & & $\begin{array}{l}\mathrm{I} \\
1\end{array}$ & $\mathrm{C}$ & $\begin{array}{l}\mathrm{I} \\
-\end{array}$ & $\begin{array}{l}\mathrm{C} \\
-\end{array}$ & $\begin{array}{l}\mathrm{I} \\
-\end{array}$ & $\begin{array}{l}\mathrm{C} \\
-\end{array}$ & I & $\begin{array}{l}\mathrm{C} \\
-\end{array}$ & $\begin{array}{l}\text { I } \\
1\end{array}$ & $\begin{array}{l}\mathrm{C} \\
-\end{array}$ \\
\hline & \multirow{2}{*}{$\begin{array}{l}\text { No estoy } \\
\text { seguro }\end{array}$} & \multicolumn{2}{|c|}{$\begin{array}{c}18 \\
(53 \%)\end{array}$} & \multicolumn{2}{|c|}{$\begin{array}{c}3 \\
(8.9 \%)\end{array}$} & \multicolumn{2}{|c|}{$\begin{array}{c}9 \\
(26.4 \%)\end{array}$} & \multicolumn{2}{|c|}{$\begin{array}{c}3 \\
(8.9 \%)\end{array}$} & \multicolumn{2}{|c|}{$\begin{array}{c}1 \\
(3 \%)\end{array}$} \\
\hline & & $\begin{array}{c}\mathrm{I} \\
12\end{array}$ & $\begin{array}{l}C \\
6\end{array}$ & $\begin{array}{l}\text { I } \\
2\end{array}$ & $\begin{array}{l}\mathrm{C} \\
1\end{array}$ & $\begin{array}{l}\text { I } \\
9\end{array}$ & $\begin{array}{l}\mathrm{C} \\
-\end{array}$ & $\begin{array}{l}\text { I } \\
3\end{array}$ & $\begin{array}{l}\mathrm{C} \\
-\end{array}$ & $\begin{array}{l}\mathrm{I} \\
1\end{array}$ & $\begin{array}{l}\mathrm{C} \\
-\end{array}$ \\
\hline & \multirow{2}{*}{ De acuerdo } & \multicolumn{2}{|c|}{$\begin{array}{c}156 \\
(84.8 \%)\end{array}$} & \multicolumn{2}{|c|}{$\begin{array}{c}11 \\
(6 \%)\end{array}$} & \multicolumn{2}{|c|}{$\begin{array}{c}8 \\
(4.3 \%)\end{array}$} & \multicolumn{2}{|c|}{$\begin{array}{c}7 \\
(3.9 \%)\end{array}$} & \multicolumn{2}{|c|}{$\begin{array}{c}2 \\
(1 \%)\end{array}$} \\
\hline & & $\begin{array}{c}\mathrm{I} \\
105\end{array}$ & $\begin{array}{c}\mathrm{C} \\
51\end{array}$ & $\begin{array}{l}\mathrm{I} \\
8\end{array}$ & $\begin{array}{l}\mathrm{C} \\
3\end{array}$ & $\begin{array}{l}\mathrm{I} \\
8\end{array}$ & $\begin{array}{l}\mathrm{C} \\
-\end{array}$ & $\begin{array}{l}\text { I } \\
7\end{array}$ & $\begin{array}{l}\mathrm{C} \\
-\end{array}$ & $\begin{array}{l}\mathrm{I} \\
2\end{array}$ & $\begin{array}{l}\mathrm{C} \\
-\end{array}$ \\
\hline & \multirow{2}{*}{$\begin{array}{l}\text { Totalmente de } \\
\text { acuerdo }\end{array}$} & \multicolumn{2}{|c|}{$\begin{array}{c}502 \\
(85.2 \%)\end{array}$} & \multicolumn{2}{|c|}{$\begin{array}{c}42 \\
(7.1 \%)\end{array}$} & \multicolumn{2}{|c|}{$\begin{array}{c}29 \\
(5 \%)\end{array}$} & \multicolumn{2}{|c|}{$\begin{array}{c}11 \\
(1.9 \%)\end{array}$} & \multicolumn{2}{|c|}{$\begin{array}{c}5 \\
(0.9 \%)\end{array}$} \\
\hline & & $\begin{array}{c}\text { I } \\
347\end{array}$ & $\begin{array}{c}\mathrm{C} \\
155\end{array}$ & $\begin{array}{c}\mathrm{I} \\
33\end{array}$ & $\begin{array}{l}\mathrm{C} \\
9\end{array}$ & $\begin{array}{c}\mathrm{I} \\
27\end{array}$ & $\begin{array}{l}\mathrm{C} \\
2\end{array}$ & $\begin{array}{l} \\
9\end{array}$ & $\begin{array}{l}\mathrm{C} \\
2\end{array}$ & $\begin{array}{l}\text { I } \\
2\end{array}$ & $\begin{array}{l}\mathrm{C} \\
3\end{array}$ \\
\hline
\end{tabular}

Tabla 3. Optimismo de los estudiantes respecto finalización del Grado y sentimiento diferente por género. 


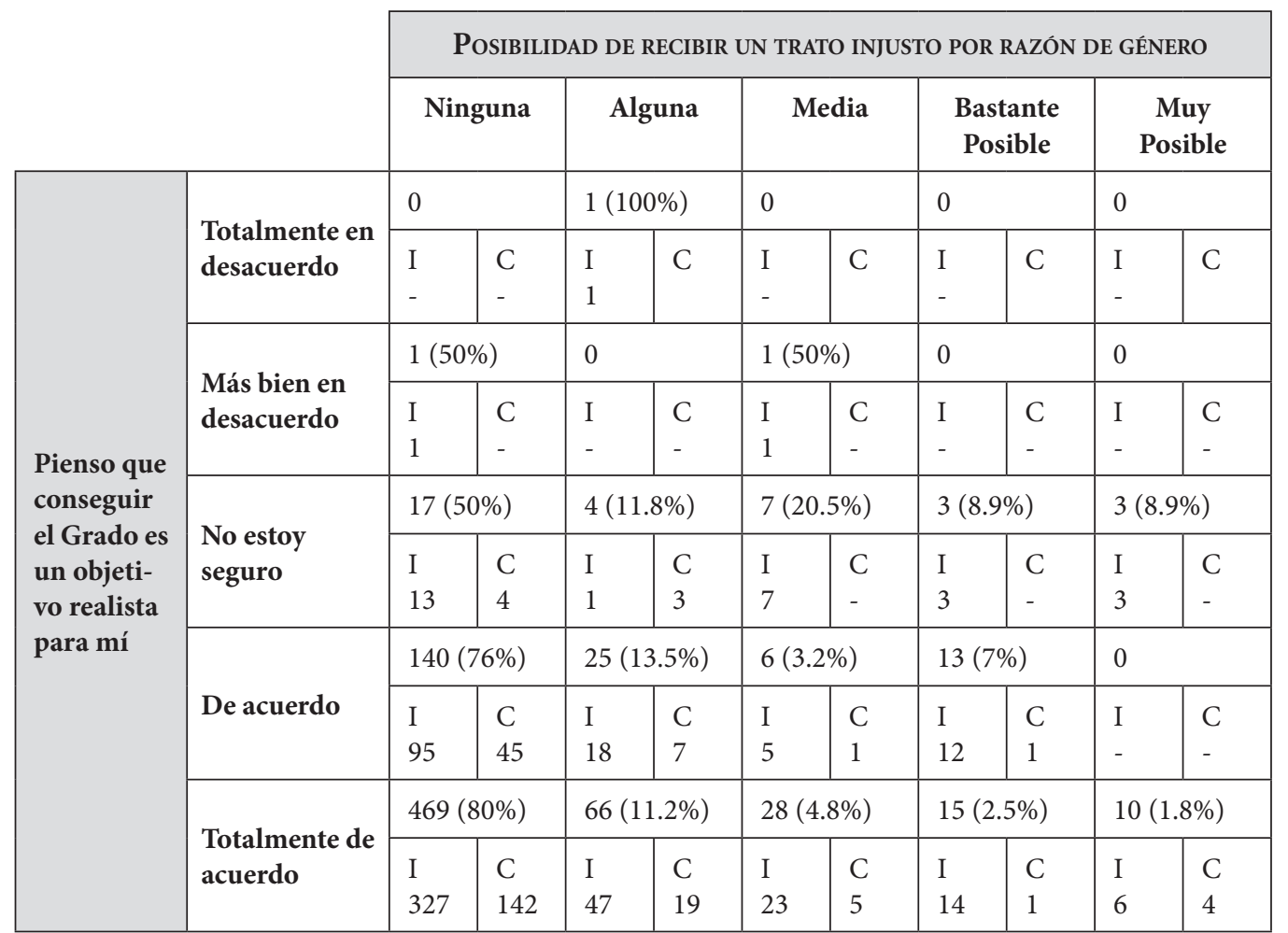

Tabla 4. Optimismo de los estudiantes respecto finalización del Grado y trato injusto por razón de género.

años de carrera (Coeficiente de Contingencia= $0.265, \mathrm{p}=0.000$ ) como puede constatarse en la Tabla 4. .

Sin embargo, en contra de lo que plantean muchos estudios no parece que ni alumnos $n$ alumnas perciban que el nivel de exigencia de los estudios vaya a interferir en sus responsabilidades familiares (cuidado de hijos, persona dependientes u otras), refrendándose en que no hay una relación estadísticamente significativa entre dos ítems del cuestionario que se incluían en el instrumento de recogida de información (Tablas 5 y 6 respectivamente ), respecto a esta dificultad de conciliación («Percibir que las demandas que supone continuar en este grado interferirán en mis responsabilidades familiares» -Coeficiente de Contingencia= $0.128, p=0.195-y$, «Estar preocupado por que esta carrera profesional requerirá mucho tiempo y formación»-Coeficiente de Contingencia $=0.125, p=0.116-$ ). Sería interesant

contrastar estos datos con sus respuestas ante estas mismas preguntas cuando estos alumnos se encuentren a punto de finalizar sus estudios universitarios y cuando, por tanto, visualicen de una manera más clara la necesidad de tomar decisiones profesionales inmediatas.

Sin embargo, y ésta es una cuestión que debería preocuparnos como docentes, inquieta su percepción acerca de las dificultades de comprensión o su impresión de tener malos docentes (Ver Tabla 7). Así, del conjunto de la muestra menos de la mitad (48.6\%) responden con rotundidad que no se han sentido perjudicados por dificultades de comprensión (categorías «Totalmente en Desacuerdo» y «Más bien en Desacuerdo»). En este caso, la situación entre los estudiantes de ingeniería y de ciencias no reviste diferencias notables. Además, un 40,2\% del conjunto de la muestra está convencido de que se encontrará profesores poco competentes en sus estudios: así lo verifican un $19,5 \%$ que

\begin{tabular}{|c|c|c|c|c|c|c|c|c|c|c|c|}
\hline & & Perc & R QU & AS DE & $\begin{array}{l}\text { IDAS } \\
\text { ESPO }\end{array}$ & $\begin{array}{l}\text { SUP } \\
\text { BILII }\end{array}$ & $\begin{array}{l}\text { E CO } \\
\text { ES F }\end{array}$ & $\begin{array}{l}\text { UAR } \\
\text { IARI }\end{array}$ & ADO & ERF & ÁN EN \\
\hline & & & & & & & & & & & osible \\
\hline \multirow{4}{*}{ Género } & \multirow[b]{2}{*}{ Femenino } & \multicolumn{2}{|c|}{$148(51.5 \%)$} & \multicolumn{2}{|c|}{$65(22.7 \%)$} & \multicolumn{2}{|c|}{$50(17.4 \%)$} & \multicolumn{2}{|c|}{$19(6.6 \%)$} & \multicolumn{2}{|c|}{$5(1.8 \%)$} \\
\hline & & $\begin{array}{c}\text { I } \\
81\end{array}$ & $\begin{array}{c}C \\
67\end{array}$ & $\begin{array}{c}\text { I } \\
37\end{array}$ & $\begin{array}{c}C \\
28\end{array}$ & $\begin{array}{c}\text { I } \\
26\end{array}$ & $\begin{array}{c}C \\
24\end{array}$ & $\begin{array}{c}\text { I } \\
14\end{array}$ & $\begin{array}{l}\mathrm{C} \\
5\end{array}$ & $\begin{array}{l}\text { I } \\
4\end{array}$ & $\begin{array}{l}\mathrm{C} \\
1\end{array}$ \\
\hline & \multirow{2}{*}{ Masculino } & \multicolumn{2}{|c|}{204 (39.3\%) } & \multicolumn{2}{|c|}{$139(26.9 \%))$} & \multicolumn{2}{|c|}{$\begin{array}{c}123 \\
(23.8 \%)\end{array}$} & \multicolumn{2}{|c|}{$\begin{array}{c}44 \\
(8,4 \%)\end{array}$} & \multicolumn{2}{|c|}{$\begin{array}{c}8 \\
(1.5 \%)\end{array}$} \\
\hline & & $\begin{array}{c}\text { I } \\
156\end{array}$ & $\begin{array}{c}C \\
48\end{array}$ & $\begin{array}{c}\mathrm{I} \\
110\end{array}$ & $\begin{array}{c}\text { C } \\
29\end{array}$ & $\begin{array}{c}\text { I } \\
104\end{array}$ & $\begin{array}{c}\text { C } \\
19\end{array}$ & $\begin{array}{c}\text { I } \\
36\end{array}$ & $\begin{array}{l}\mathrm{C} \\
8\end{array}$ & I & $\begin{array}{l}\text { C } \\
1\end{array}$ \\
\hline
\end{tabular}

Tabla 5. Interferencia de los estudios con responsabilidades familiares y género.

\begin{tabular}{|c|c|c|c|c|c|c|c|c|c|c|c|}
\hline & \multicolumn{10}{|c|}{$\begin{array}{l}\text { ESTAR PREOCUPADO PORQUE ESTA CARRERA PROFESIONAL REQUERIRÁ MUCHO } \\
\text { TIEMPO Y FORMACIÓN }\end{array}$} \\
\hline & & \multirow{2}{*}{\multicolumn{2}{|c|}{$\begin{array}{l}\text { Ninguna } \\
28(9.7 \%)\end{array}$}} & \multirow{2}{*}{\multicolumn{2}{|c|}{$\begin{array}{c}\text { Alguna } \\
44(15.2 \%)\end{array}$}} & \multirow{2}{*}{\multicolumn{2}{|c|}{$\begin{array}{c}\text { Media } \\
92(31.9 \%)\end{array}$}} & \multirow{2}{*}{\multicolumn{2}{|c|}{$\begin{array}{c}\begin{array}{c}\text { Bastante } \\
\text { Posible }\end{array} \\
90(31.1 \%)\end{array}$}} & \multirow{2}{*}{\multicolumn{2}{|c|}{\begin{tabular}{|l} 
Muy Posible \\
$35(12.1 \%)$
\end{tabular}}} \\
\hline \multirow{4}{*}{ Género } & \multirow[b]{2}{*}{ Femenino } & & & & & & & & & & \\
\hline & & $\begin{array}{c}\text { I } \\
13\end{array}$ & $\begin{array}{c}\mathrm{C} \\
15\end{array}$ & $\begin{array}{c}\mathrm{I} \\
16\end{array}$ & $\begin{array}{l}\mathrm{C} \\
28\end{array}$ & $\begin{array}{c}\text { I } \\
62\end{array}$ & $\begin{array}{c}\mathrm{C} \\
30\end{array}$ & $\begin{array}{c}\text { I } \\
54\end{array}$ & $\begin{array}{l}\mathrm{C} \\
36\end{array}$ & $\begin{array}{c}\mathrm{I} \\
18\end{array}$ & $\begin{array}{c}\mathrm{C} \\
17\end{array}$ \\
\hline & \multirow[b]{2}{*}{ Masculino } & \multicolumn{2}{|c|}{$73(14 \%)$} & \multicolumn{2}{|c|}{$112(21.6 \%)$} & \multicolumn{2}{|c|}{$158(30.5 \%)$} & \multicolumn{2}{|c|}{$126(24.3 \%)$} & \multicolumn{2}{|c|}{$49(9,4 \%)$} \\
\hline & & $\begin{array}{c}\text { I } \\
57\end{array}$ & $\begin{array}{c}\mathrm{C} \\
16\end{array}$ & $\begin{array}{l}\text { I } \\
91\end{array}$ & $\begin{array}{l}\mathrm{C} \\
21\end{array}$ & $\begin{array}{c}\mathrm{I} \\
122\end{array}$ & $\begin{array}{l}\mathrm{C} \\
36\end{array}$ & $\begin{array}{c}\mathrm{I} \\
102\end{array}$ & $\begin{array}{l}\mathrm{C} \\
24\end{array}$ & $\begin{array}{c}\mathrm{I} \\
41\end{array}$ & $\begin{array}{l}\mathrm{C} \\
8\end{array}$ \\
\hline
\end{tabular}

Tabla 6. Preocupación por el tiempo y formación que requerirá carrera profesional y género

\begin{tabular}{|c|c|c|c|c|c|c|c|c|c|c|}
\hline & \multicolumn{2}{|c|}{$\begin{array}{l}\text { TOtalmente en } \\
\text { Desacuerdo }\end{array}$} & \multicolumn{2}{|c|}{$\begin{array}{l}\text { Más biEn en } \\
\text { DesaCuerdo }\end{array}$} & \multicolumn{2}{|c|}{$\begin{array}{l}\text { No estoy } \\
\text { SEgURO }\end{array}$} & \multicolumn{2}{|c|}{ De ACuerdo } & \multicolumn{2}{|c|}{$\begin{array}{l}\text { Totalmente } \\
\text { DE ACuerdo }\end{array}$} \\
\hline \multirow{2}{*}{$\begin{array}{l}\text { Me he sentido } \\
\text { perjudicado por } \\
\text { dificultades de } \\
\text { comprensión, } \\
\text { docentes difíciles } \\
\text { de entender }\end{array}$} & \multicolumn{2}{|c|}{$153(18.8 \%)$} & \multicolumn{2}{|c|}{$242(29.8 \%)$} & \multicolumn{2}{|c|}{$197(24.3 \%)$} & \multicolumn{2}{|c|}{$146(18 \%)$} & \multicolumn{2}{|c|}{$72(8.9 \%)$} \\
\hline & $\begin{array}{c}\text { I } \\
104\end{array}$ & $\begin{array}{c}\mathrm{C} \\
49\end{array}$ & $\begin{array}{c}\text { I } \\
176\end{array}$ & $\begin{array}{l}\mathrm{C} \\
66\end{array}$ & $\begin{array}{c}\mathrm{I} \\
128\end{array}$ & $\begin{array}{l}\mathrm{C} \\
69\end{array}$ & $\begin{array}{c}\mathrm{I} \\
111\end{array}$ & $\begin{array}{l}\mathrm{C} \\
35\end{array}$ & $\begin{array}{c}\text { I } \\
59\end{array}$ & $\begin{array}{l}\mathrm{C} \\
13\end{array}$ \\
\hline \multirow{2}{*}{$\begin{array}{l}\text { Creo que tendré } \\
\text { posibilidades } \\
\text { de encontrarme } \\
\text { malos docentes en } \\
\text { las asignaturas de } \\
\text { la carrera }\end{array}$} & \multicolumn{2}{|c|}{$62(7.6 \%)$} & \multicolumn{2}{|c|}{$210(25.9 \%)$} & \multicolumn{2}{|c|}{$212(26.1 \%)$} & \multicolumn{2}{|c|}{168 (20.7\%) } & \multicolumn{2}{|c|}{$158(19.5 \%)$} \\
\hline & $\begin{array}{c}\text { I } \\
38\end{array}$ & $\begin{array}{l}C \\
24\end{array}$ & $\begin{array}{c}\text { I } \\
141\end{array}$ & $\begin{array}{l}\mathrm{C} \\
69\end{array}$ & $\begin{array}{c}\text { I } \\
146\end{array}$ & $\begin{array}{l}C \\
66\end{array}$ & $\begin{array}{c}\mathrm{I} \\
125\end{array}$ & $\begin{array}{c}C \\
43\end{array}$ & $\begin{array}{c}\mathrm{I} \\
128\end{array}$ & C 30 \\
\hline
\end{tabular}

Tabla 7. Opinión de los estudiantes respecto a dificultades académicas. 
sostienen estar «Totalmente de acuerdo» con esta afirmación y un 20,7\% que se ubican en la categoría «De acuerdo».

Ante estas dificultades podría ser de utilidad disponer de algún tipo de asesoramiento profesional. En efecto, la orientación educativa y profesional ya no se concibe en la actualidad como un proceso terminal que finaliza en el momento en el que se decide la opción de estudios postsecundarios que se va a realizar sino que se defiende una concepción mucho más amplia, que se desarrollará a lo largo de toda la vida y que cristaliza en los Planes de Acción Tutorial que la práctica totalidad de los centros han establecido como medidas de apoyo al proceso de Bolonia. Sorprenden por tanto las dudas de los estudiantes que se ubican en las categorías «Media» (30.7\%), «Alguna (8.9\%) y «Ninguna» (3.4\%) ante la pregunt que se centra en la posibilidad de recibir ayuda de un tutor/a en caso de necesitarlo (ver Tabla 8). En esta ocasión, parecen ser los estudiante de ingenierías los más pesimistas o los peor informados acerca de la presencia de tutores académicos o planes de acción tutorial que pueden contribuir a solventar sus indecisiones.

No obstante, como se refleja en la Tabla 8 resultan mucho más altos los porcentajes de estudiantes que consideran que no tendrán dificultades en recibir ayuda de otra posible fuente de información, sus docentes: el 20.3\% se posicionan en la respuesta «Ninguna di-

ficultad» y el $34.9 \%$ en «Alguna dificultad». Nuevamente, la percepción de los estudiantes de Ciencias parece más optimista que la de los estudiantes de ingeniería.

\section{Conclusiones}

Los estudiantes encuestados han mostrado tener importantes creencias de autoeficacia, reflejándose esta percepción en su confianza en continuar matriculados durante el curso académico en el que están actualmente así como, en un futuro más lejano, su convencimiento de que finalizarán los estudios de Grado que han iniciado. Los datos reflejan cómo los estudiantes de ingenierías obtienen unas puntuaciones más bajas que los estudiantes de ciencias en ambos ítems. En momentos posteriores de la investigación será necesario revisar si este optimismo se mantiene a lo largo del curso académico, cuando los resultados de los exámenes permitan tener quizás datos más objetivos del rendimiento que se está obteniendo (el cuestionario ha sido administrado durante el primer semestre).

De manera general no parece que ni la edad ni el género influyan en las respuestas a estas dos preguntas (continuar matriculado el semestre y finalizar los estudios. Además, la convicción de que finalizarán los estudios mantiene una relación estadísticamente significativa con haberse sentido diferente por motivo de

\begin{tabular}{|c|c|c|c|c|c|c|c|c|c|c|}
\hline \multirow{3}{*}{$\begin{array}{l}\text { Conseguir } \\
\text { ayuda un } \\
\text { tutor/a en } \\
\text { caso de que lo } \\
\text { necesitase }\end{array}$} & \multicolumn{2}{|c|}{ Ninguna } & \multicolumn{2}{|c|}{ AlguNa } & \multicolumn{2}{|c|}{ Media } & \multicolumn{2}{|c|}{$\begin{array}{c}\text { BASTANTE } \\
\text { POSIBLE }\end{array}$} & \multicolumn{2}{|c|}{ MuY POSIBLE } \\
\hline & \multicolumn{2}{|c|}{$28(3.4 \%)$} & \multicolumn{2}{|c|}{$72(8.9 \%)$} & \multicolumn{2}{|c|}{$249(30.7 \%)$} & \multicolumn{2}{|c|}{$307(37.8 \%)$} & \multicolumn{2}{|c|}{$156(19.2 \%)$} \\
\hline & $\begin{array}{c}I \\
23\end{array}$ & $\begin{array}{l}\mathrm{C} \\
5\end{array}$ & $\begin{array}{c}\mathrm{I} \\
55\end{array}$ & $\begin{array}{c}\mathrm{C} \\
17\end{array}$ & $\begin{array}{c}\text { I } \\
192\end{array}$ & $\begin{array}{l}\text { C } \\
57\end{array}$ & $\begin{array}{c}\mathrm{I} \\
210\end{array}$ & $\begin{array}{l}\mathrm{C} \\
97\end{array}$ & $\begin{array}{c}\mathrm{I} \\
100\end{array}$ & $\begin{array}{c}\mathrm{C} \\
56\end{array}$ \\
\hline \multirow{2}{*}{$\begin{array}{l}\text { Encontrar } \\
\text { dificultades } \\
\text { para recibir } \\
\text { ayuda de los/ } \\
\text { las docentes }\end{array}$} & \multicolumn{2}{|c|}{$165(20.3 \%)$} & \multicolumn{2}{|c|}{$283(34.9 \%)$} & \multicolumn{2}{|c|}{$261(32.1 \%)$} & \multicolumn{2}{|c|}{$65(8 \%)$} & \multicolumn{2}{|c|}{$36(4.4 \%)$} \\
\hline & $\begin{array}{c}\mathrm{I} \\
94\end{array}$ & $\begin{array}{c}\mathrm{C} \\
71\end{array}$ & $\begin{array}{c}\text { I } \\
189\end{array}$ & $\begin{array}{l}\mathrm{C} \\
94\end{array}$ & $\begin{array}{c}\text { I } \\
207\end{array}$ & $\begin{array}{c}\mathrm{C} \\
54\end{array}$ & $\begin{array}{c}\text { I } \\
55\end{array}$ & $\begin{array}{c}\mathrm{C} \\
10\end{array}$ & $\begin{array}{c}\mathrm{I} \\
33\end{array}$ & $\begin{array}{l}\mathrm{C} \\
3\end{array}$ \\
\hline
\end{tabular}

Tabla 8. Ayudas y apoyos en el centro. género y manifestar que han recibido durante los primero años de carrera un trato injusto po razón de género. Estos datos refrendarían lo obtenidos en otros estudios (Erwin y Maurutto 1998; Rodd y Bartholomew, 2006) en los que las alumnas de estudios científicos manifestaban sentirse invisibles o ser tratadas de maner desigual por determinados profesores: no escucha de sus respuestas, descripción como mejo alumno de la clase referida a un varón a pesa de que el resultado académico más brillante sea el de una chica, profesores que se desplazan por el aula únicamente hacia el lugar ocupado po los chicos, la preconcepción de que la capacidad de las alumnas es innatamente menor, etc. Además, estos comportamientos son mucho más frecuentes en las carreras de tipo científico tecnológico -aquéllas en las que hemos centrado nuestro estudio- que en las del ámbito de la ciencias sociales o humanidades, en las que la presencia femenina tanto de docentes como de alumnas tiene una mayor trayectoria y que responde, en cierta medida, a la creencia "natural" de la habilidad de hombres y mujeres para según qué estudios.

Parece conveniente destacar que los estudiantes no perciben, al menos en la actualidad, que puedan existir interferencias entre el nive de exigencia de los estudios y sus responsabilidades familiares. Ésta será otra cuestión que convendrá explorar en la fase cualitativa de la investigación para corroborar si han hecho una estimación de la incidencia en el futuro o incluso, si esta negativa, sería el efecto directo de que en la actualidad no poseen ningún tipo de cargas familiares. En suma, sorprende que su percepción parece contradecir la denuncia de muchos de los estudios realizados sobre este tema, a saber, las dificultades para la conciliación y más particularmente en el caso de las mujeres (Bermúdez et al., 2011; Blättel-Mink, 2002; Donoso et al., 2011; Erwin y Maurutto 1998 ;Erwin y Stewart, 1997; Folch y Ramos, 2009; García-Gómez et al., 2009; Jackson, 2000 Alonso y Mas, 2009; Sax y Harper, 2007)

En el plano más propiamente pedagógico conviene resaltar los resultados referidos a un de los puntales de la variable denominada "per- suasión y apoyo social»: el soporte que creen poder recibir de docentes y tutores. En el caso de los docentes, aunque muchos de ellos consideran que las probabilidades de encontrarse a lo largo de sus estudios con «malos» docentes es importante, sin embargo, consideran mayoritariamente que no tendrán dificultades en conseguir ayuda de ellos en caso de necesitara. Convendría por tanto clarificar qué están entendiendo exactamente por malos docentes: aquellos cuyo nivel de exigencia es alto, profesores que resultan incomprensibles, docentes cuyas clases no resultan interesantes, etc. Sobre este particular, las respuestas de los estudiantes de ciencias resultan ligeramente más optimistas.

Finalmente, se concluye que un número importante de estudiantes desconoce la existencia de tutores, figura que quedaría enmarcada en Planes de Acción Tutorial que han incluido la mayoría de los centros en sus memorias de verificación para los nuevos Grados y que, aunque poco frecuente en el contexto español, tiene una larga trayectoria histórica en otros sistemas educativos. Esta desinformación preocupa de manera especial en tanto en cuanto más de la mitad del alumnado encuestado admite que tienen o considera que tendrá importantes dificultades de comprensión y por lo tanto, parece previsible que precisen algún tipo de apoyo extra como aprendices adultos.

\section{REFERENCIAS}

Alonso, A. V. y Mas, M. A. M. (2009). Patrones actitudinales de la vocación científica y tecnológica en chicas y chicos de Secundaria. Revista Iberoamericana de Educación, 50(4), 1-12. Recuperado enero 17, 2013 de http://www. rieoei.org/deloslectores/2950Vazquez.pdf.

Ayalon, H. (2003). Women and men go to University: mathematical background and gender differences in choice of field in Higher Education. Sex Roles, 48(5-6), 277-290.

Bandura, A., Barbaranelli, C., Caprara, G. y Pastorelli, C. (2001). Self-efficacy beliefs as shapers of children's aspirations and career trajectories. Child Development, 72(1), 187-206. 
Bermúdez, M. P., Guillén-Riquelme, A., GómezGarcía, A., Quevedo-Blasco, R., Sierra, J. C. y Buela-Casal, G. (2011). Análisis del rendimiento en el doctorado en función del sexo. Educación XXI, 14(1), 17-33.

Betz, N. E. y Borgen, F. H. (2000). The future of career assessment: integrating vocational interest with self-efficacy and personal styles. Journal of Career Assessment, 8(4), 329-338.

Betz, N. E. y Hackett, G. (1981). The relationship of career-related self-efficacy expectations to perceived career options in college women and men. Journal of Counseling Psychology, 28 (5), 399-410.

Betz, N. E. y Hackett, G. (1997). Applications of self-efficacy theory to the career assessment of women. Journal of Career Assessment, 5(4), 383-402.

Betz, N. E. y Hackett, G. (2006). Career self-efficacy theory: back to the future. Journal of $\mathrm{Ca}$ reer Assessment, 14 (3), 3-11.

Betz, N. y Schifano, R. (2000). Evaluation of an intervention to increase realistic self-efficacy and interests in college women. Journal of Vocational Behavior, 56 (1), 35-52.

Blättel-Mink, B. (2002). Gender and subject decision at University. Gender specificity in subject perception and decision with main emphasis on science and technology. Equal Opportunities International, 21 (1), 43-64.

Britner, S. y Pajares, F. (2006). Sources of science self-efficacy beliefs of middle school students. Journal of Research in Science Teaching, 43(5) 485-499.

Donoso, T., Figuera, P. y Rodríguez-Moreno, M L. (2011). Barreras de género en el desarrollo profesional de la mujer universitaria. Revista de Educación, 355, 187- 212.

Erwin, L. y Mauruttto, P. (1998). Beyond access: considering gender deficits in science education. Gender and Education, 10(1), 51-69.

Erwin, L. y Stewart, P. (1997). Gendered perspectives: a focus - group study of how undergraduate women negotiate their career aspirations. Qualitative studies in education, 10 (2) 207-220.

Evers, F., Livernois, J. y Mancuso, M. (2006) Where are the boys? Gender imbalance in Higher Education. Higher Education Management and Policy, 18(2), 1-13.
Feldman, R. S. (2005) Improving the first year of college: research and practice. Mahwah: Lawrence Erlbaum Associates.

Fernández, C. M. G., Peña, J. V. C., Viñuela, M. P. H. y Torío, S. L. (2007). Los procesos de orientación escolar y la toma de decisiones académica y profesional. Revista Complutense de Educación, 18 (2), 87-103.

Fernández, C. M. G., Viñuela, M. P. H., Peña, J. V. C. y Molina, S. M. (2006). El peso del sistema educativo sobre las expectativas académicas y profesionales de los adolescentes. Revista de Investigación Educativa, 24 (2), 475-495.

Folch, M. T. y Ramos, C. G. (2009). Las barreras y los obstáculos en el acceso de las profesoras universitarias a los cargos de gestión académica. Revista de Educación, 350, 253 - 275.

García-Gómez, S., Padilla-Carmona, M. T. y Suárez-Ortega, M. (2009). Los intereses académicos y profesionales de chicas que finalizan la escolaridad obligatoria. Revista de Educación, 349, 311-334.Guimond, S. y Roussel, L. (2001). Bragging about one's school grades: gender stereotyping and students' perception of their abilities in science, mathematics and language. Social Psychology of Education, 4(34), 275-293.

Hackett, G. (1985). Role of mathematics selfefficacy in the choice of math-related majors of college women and men: a path analysis. Journal of Counseling Psychology, 32(1), 47-56. Hackett, G. y Betz, N. E. (1981). A self-efficacy approach to the career development of women. Journal of Vocational Behavior, 18(3), 326-339.

Jackson, S. (2000). Differently academic? Constructions of 'academic' in higher education. Higher education Research and Development, 19(3), 279-296.

Lent, R. W., López, F. G., Brown, S. D. y Gore, P. A. (1996). Latent structure of the sources of mathematics self-efficacy. Journal of Vocational Behavior, 49 (3), 292-308.

López, S. T., García, J. H. y Calvo, J. V. P. (2007). Capital social familiar y expectativas académico-formativas y laborales del alumnado de Educación Secundaria Obligatoria. Revista de Educación, 343, 559-586.

Marbá-Tallada, A. y Solsona-Pairó, N. (2012). Identificación e interpretación e las posi- bles desigualdades formativas en ciencias de chicos y chicas en la educación obligatoria y el bachillerato. Cultura y educación, 24(3), 289-303.

Martín-Del Buey, F. y Camarero-Suárez, F. (2001). Diferencias de género en los procesos de aprendizaje en universitarios. Psicothema 13(4), 598-604.

Miura, I. T. (1987). The relationship of computer self-efficacy expectations to computer interes and course enrollment in college. Sex Roles, $16(5 / 6), 303-311$.

Olaz, F. O. (2003). Autoeficacia y diferencia de géneros. Aportes a la explicación del comportamiento vocacional. Revista de Psicología General y Aplicada, 56 (3), 359-376.

Pajares, F. (2005). Gender differences in mathematics self-efficacy beliefs. En Gallagher A. M. y Kaufman J. C. (Eds.). Gender differences in mathematics. An integrative psychologi cal approach (pp. 294-315). New York: Cambridge University Press.

Rego, M. A. S. y Castro, A. M. P. (2002). Género valores y orientación vocacional en un contexto universitario para la salud, reto de nuestro tiempo. Educación XXI, 5(1), 19-34.

Rodd, M. y Bartholomew, H. (2006). Invisible and special: young women's experiences as undergraduate mathematic students. Gender and Education, 18(1), 35-50.
Rodríguez-Menéndez, M. C., Inda-Caro, M. y Peña-Calvo, J. V. (2015). Validación de la teoría cognitivo social de desarrollo de la carrera con una muestra de estudiantes de ingeniería. Educación XXI, 18(2), p. 1-26 (en prensa).

Rodríguez-Menéndez, M. C., Torío-López, S. y Fernández-García, C. M. (2006). El impacto del género en las elecciones académicas de los estudiantes asturianos que finalizan $4^{\circ}$ de la ESO. Revista Española de Orientación y Psicopedagogía, 17 (2), 239-260.

Sax, L. J. y Harper, C. (2007). Origins of the gender gap: pre - college and college influences on differences between men and women. Research in Higher Education, 48(6), 669-694.

Vouillot, F., Blanchard, S., Marro, C. y Steinbruckner, M-L. (2004). La division sexuée de lorientation et du travail: une question théorique et une question de pratiques. Psychologie du Travail et des Organisations, 10, 277-291.

Zeldin, A. L. y Pajares, F. (2000). Against the odds: self-efficacy beliefs of women in mathematical, scientific and technological careers. American Educational Research Journal, 37(1), 215-246.

Zeldin, A. L., Britner, S. L. y Pajares, F. (2008). A comparative study of the self-efficacy beliefs of successful men and women in mathematics, science and technology careers. Journal of Research in Science Teaching, 45(9), 1036-1058. 
